Article

\title{
The Maximum Hosoya Index of Unicyclic Graphs with Diameter at Most Four
}

\author{
Weijun Liu ${ }^{1,+}{ }^{\dagger}$ Jingwen Ban ${ }^{1, \dagger}$, Lihua Feng ${ }^{1, \dagger}{ }^{+}$, Tao Cheng ${ }^{2,+}$, Frank Emmert-Streib ${ }^{3, \dagger}$ and \\ Matthias Dehmer $4,5,6, *,+$ \\ 1 School of Mathematics and Statistics, Central South University New Campus, Changsha 410083, China \\ 2 School of Mathematics and Statistics, Shandong Normal University, Jinan 250014, China \\ 3 Predictive Society and Data Analytics Lab Faculty of Information Technolgy and Communication Sciences, \\ Tampere University, 33100 Tampere, Finland \\ 4 School of Management, University of Applied Sciences Upper Austria, 4400 Steyr, Austria \\ 5 Department of Biomedical Computer Science and Mechatronics UMIT, 6060 Hall in Tyrol, Austria \\ 6 College of Articial Intelligence, Nankai University, Tianjin 300350, China \\ * Correspondence: matthias.dehmer@umit.at \\ + These authors contributed equally to this work.
}

Received: 28 June 2019; Accepted: 6 August 2019; Published: 9 August 2019

check for updates

\begin{abstract}
The Hosoya index of a graph is defined by the total number of the matchings of the graph. In this paper, we determine the maximum Hosoya index of unicyclic graphs with $n$ vertices and diameter 3 or 4 . Our results somewhat answer a question proposed by Wagner and Gutman in 2010 for unicyclic graphs with small diameter.
\end{abstract}

Keywords: Hosoya index; unicyclic graphs; diameter

\section{Introduction}

Numerous topological and chemical indices/measures have been used for analyzing molecular graphs [1-4]. A prominent example is the Hosoya index introduced by Hosoya [5] in 1971 as a molecular-graph based structure descriptor. Hosoya discovered that certain physico-chemical properties of alkanes (= saturated hydrocarbons) —in particular, their boiling points-are well correlated with this index. Gutman et al. further considered it in the chemical view [6]. As is known, structural graph descriptors have been investigated extensively in chemistry, drug design and related disciplines [1-4].

The Hosoya index got much attention by many researchers in the past decades. They have been interested in identifying the maximum or minimum value of Hosoya index for various classes of graphs (with certain restrictions), such as trees [7-9], unicyclic graphs [10-14], bicyclic graphs [15] and so on. For an exhaustive survey for this topic, we refer to [16].

Even though there is a considerable amount of literature on the topic of maximizing or minimizing the Hosoya index, there are still many interesting open questions left. In [16], it is mentioned that:

- It seems to be difficult to obtain results of the maximum Hosoya index among trees with a given number of leaves or given diameter. However, partial results are available, so the problem might not be totally intractable, and results in this direction would definitely be interesting.

- If the aforementioned questions can be answered for trees, then it is also natural to consider the analogous questions for treelike graphs (such as unicyclic graphs).

For two vertices $u, v$ in a graph $G$, the distance $d(u, v)$ between $u$ and $v$ is the length of a shortest path connecting them. The diameter of $G$ is $\max \{d(u, v) \mid u, v \in V(G)\}$. Confirming a 
conjecture proposed by $\mathrm{Ou}$ [12], Liu [8] considered the maximum Hosoya index of trees with diameter 4. Motivated by this line of research, we here consider the maximal Hosoya index of unicyclic graphs with small diameter. It seems that unicyclic graphs are only one more edge than trees, however, some of their properties change drastically such as the girth.

At the end of this section, we define some notation as well as some preliminary results that we frequently use in the sequel.

Let $G$ be a simple connected graph with vertex set $V(G)$. For $u \in V(G)$, we denote its neighborhood by $N_{G}(u)$, and denote $d_{G}(u):=N_{G}(u)$. A pendent vertex is a vertex of degree 1 . For two vertices $u_{1}$ and $u_{2}$, the distance between $u_{1}$ and $u_{2}$ is the number of edges in a shortest path joining $u_{1}$ and $u_{2}$. We use $G-u$ to denote the graph that arises from $G$ by deleting the vertex $u \in V(G)$. For other undefined notations, we refer to [17].

Given a molecular graph $G$, let $m(G, k)$ be the number of $k$ matchings of $G$. It would be convenient to define $m(G, 0)=1, m(G, 1)=e(G)$. The Hosoya index $z=z(G)$ is defined as the number of subsets of $E(G)$ in which no edges are incident, in other words, the total number of the matchings of the graph $G$. Then,

$$
z(G)=\sum_{k \geq 0} m(G, k)
$$

For the star $K_{1, p}$ of order $p+1$, when $k \geq 2$, we have $m(G, k)=0$. Then, $z\left(K_{1, p}\right)=\sum_{k \geq 0} m(G, k)=$ $m(G, 0)+m(G, 1)=1+p$.

The double star $S_{p, q}$ is a tree of order $n$ obtained from $K_{1, p}$ and $K_{1, q-1}$, by identifying a pendent vertex of $K_{1, p}$ with the center of $K_{1, q-1}$, where $p+q=n$. For $S_{p, q}$, when $k \geq 3$, we have $m(G, k)=0$, therefore

$$
\begin{aligned}
z\left(S_{p, q}\right) & =\sum_{k \geq 0} m(G, k)=m(G, 0)+m(G, 1)+m(G, 2) \\
& =1+(p+q-1)+(p-1)(q-1)=p q+1 .
\end{aligned}
$$

The following two lemmas are needed in this paper, which can be found on page 337 of [16].

Lemma 1. Let $G$ be a graph and $v$ be a vertex of $G$. Then,

$$
z(G)=z(G-v)+\sum_{u \in N_{G}(v)} z(G-\{u, v\}) .
$$

Lemma 2. If $G_{1}, G_{2}, \ldots, G_{t}$ are the components of a graph $G$, then

$$
z(G)=\Pi_{i=1}^{t} z\left(G_{i}\right)
$$

For $n \geq 6$, the unique unicyclic graph with diameter two is obtained from the star $K_{1, n-1}$ by adding an edge. For unicyclic graphs with diameter at least 5 , things become more complicated, and we believe more techniques are needed. Thus, we only consider the cases for diameter 3 and 4 . In Section 2, we determine the maximal Hosoya index of unicyclic graphs with $n$ vertices and diameter 3 (see Theorem 5). In Section 3, we determine the maximal Hosoya index of unicyclic graphs with $n$ vertices and diameter 4 (see Theorem 15).

\section{The Unicyclic Graphs with Diameter 3}

In this section, we study the maximal Hosoya index of unicyclic graphs with $n$ vertices and diameter 3 .

Let $\mathcal{U}_{n}^{3}$ be the set of all unicyclic graphs with $n$ vertices and diameter 3 . According to the length of the unique cycle and the distribution of other vertices, we may classify all the members in $\mathcal{U}_{n}^{3}$. Let $\mathcal{U}_{i}$ 
be the set of unicyclic graph of the form $G_{i}, i=1,2,3,4$. It is easy to see that the graphs from $\mathcal{U}_{1}, \mathcal{U}_{2}$, $\mathcal{U}_{3}$, and $\mathcal{U}_{4}$, and $C_{5}, C_{6}$, and $C_{7}$ are all unicyclic graphs with diameter 3 .

Let $\mathcal{U}_{1}$ be the set of unicyclic graphs of the form $G_{1}$ (as depicted in Figure 1), where $a+b+c=n$, $a, b, c \geq 1$ and at least two of $a, b, c$ are greater than 2 . Let $G_{1}^{*}$ be the graph of the form $G_{1}$ satisfying $a, b, c$ almost equal (hereafter "almost equal" means the difference of any two numbers is at most one).
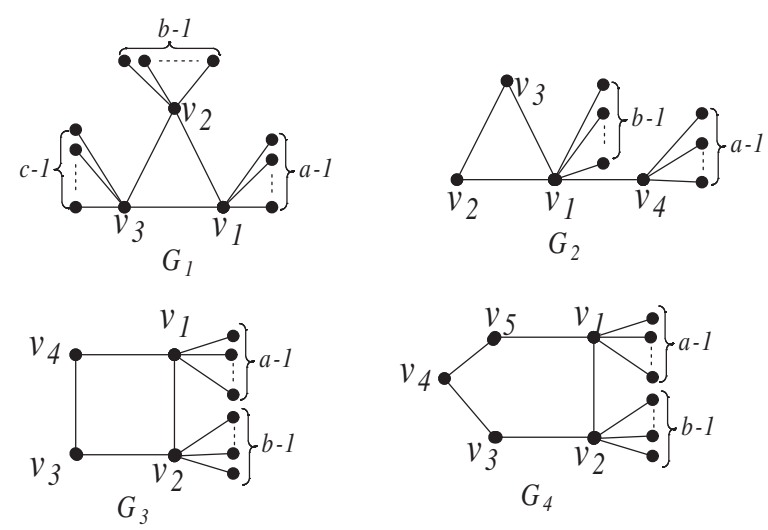

Figure 1. Four unicyclic graphs with diameter 3.

Theorem 1. The graph $G_{1}^{*}$ has the maximum Hosoya index among all graphs in $\mathcal{U}_{1}$.

Proof. Assume $G_{1} \in \mathcal{U}_{1}$ with $1 \leq a \leq b \leq c$. By Lemmas 1 and 2, we obtain

$$
\begin{aligned}
z\left(G_{1}\right) & =z\left(G_{1}-v_{1}\right)+\sum_{w \in N_{G_{1}}\left(v_{1}\right)} z\left(G_{1}-\left\{v_{1}, w\right\}\right) \\
& =z\left(G_{1}-v_{1}\right)+z\left(G_{1}-\left\{v_{1}, v_{2}\right\}\right)+z\left(G_{1}-\left\{v_{1}, v_{3}\right\}\right) \\
& +(a-1) z\left(G_{1}-\left\{v_{1}, y\right\}\right) \\
& =(b c+1)+c+b+(a-1)(b c+1) \\
& =a b c+a+b+c:=f_{1}(a, b, c)
\end{aligned}
$$

where $y$ is one of pendent vertex adjacent to $v_{1}$ in $G_{1}$.

If $b-a \geq 2$, then we get

$$
f_{1}(a+1, b-1, c)-f_{1}(a, b, c)=(b-a-1) c>0 .
$$

As $a, b, c$ have the same status as shown in the graph, we conclude that, when $a, b, c$ are almost equal, $G_{1}$ has the maximal Hosoya index.

Let $\mathcal{U}_{2}$ be the set of unicyclic graphs of the form $G_{2}$ (as depicted in Figure 1), where $a+b=n-2$, $a \geq 2, b \geq 1$. Let $G_{2}^{*}$ be the graph of the form $G_{2}$ satisfying $0 \leq a-b \leq 2$.

Theorem 2. The graph $G_{2}^{*}$ has the maximum Hosoya index among all graphs in $\mathcal{U}_{2}$.

Proof. Assume $G_{2} \in \mathcal{U}_{2}$. By Lemmas 1 and 2, we obtain

$$
\begin{aligned}
z\left(G_{2}\right) & =z\left(G_{2}-v_{1}\right)+\sum_{v^{\prime} \in N_{G_{2}}\left(v_{1}\right)} z\left(G_{2}-\left\{v_{1}, v^{\prime}\right\}\right) \\
& =z\left(G_{2}-v_{1}\right)+z\left(G_{2}-\left\{v_{1}, v_{2}\right\}\right)+z\left(G_{2}-\left\{v_{1}, v_{3}\right\}\right) \\
& +z\left(G_{2}-\left\{v_{1}, v_{4}\right\}\right)+(b-1) z\left(G_{2}-\left\{v_{1}, y\right\}\right) \\
& =2 a+a+a+2+2 a(b-1) \\
& =2 a b+2 a+2:=f_{2}(a, b)
\end{aligned}
$$


where $y$ is one of pendent vertexs adjacent to $v_{1}$ in $G_{2}$.

If $b-a \geq 1$, then we get

$$
f_{2}(a+1, b-1)-f_{2}(a, b)=2(b-a)>0 .
$$

If $a-b \geq 3$, then we get

$$
f_{2}(a-1, b+1)-f_{2}(a, b)=2(a-b-2)>0 .
$$

Thus, we obtain the result.

Let $\mathcal{U}_{3}$ be the set of unicyclic graph of the form $G_{3}$ (as depicted in Figure 1), where $v_{1}$ and $v_{2}$ are two vertices with $a-1, b-1$ pendent vertices satisfying $a+b=n-2, a, b \geq 1$, one of $a$ and $b$ is at least 2. Let $G_{3}^{*}$ be the graph of the form $G_{3}$ satisfying $a, b$ almost equal.

Theorem 3. The graph $G_{3}^{*}$ has the maximum Hosoya index among all graphs in $\mathcal{U}_{3}$.

Proof. For $G_{3} \in \mathcal{U}_{3}$ with $a \geq b \geq 1$, by Lemmas 1 and 2, we obtain

$$
\begin{aligned}
z\left(G_{3}\right) & =z\left(G_{3}-v_{1}\right)+\sum_{v^{\prime} \in N_{G_{3}}\left(v_{1}\right)} z\left(G_{3}-\left\{v_{1}, v^{\prime}\right\}\right) \\
& =z\left(G_{3}-v_{1}\right)+z\left(G_{3}-\left\{v_{1}, v_{2}\right\}\right)+z\left(G_{3}-\left\{v_{1}, v_{4}\right\}\right) \\
& +(a-1) z\left(G_{3}-\left\{v_{1}, y\right\}\right) \\
& =(2 b+1)+2+(b+1)+(a-1)(2 b+1) \\
& =2 a b+a+b+3:=f_{3}(a, b),
\end{aligned}
$$

where $y$ is one of pendent vertex adjacent to $v_{1}$ in $G_{3}$.

If $a-b \geq 2$, then we get

$$
f_{3}(a-1, b+1)-f_{3}(a, b)=2(a-b-1)>0 .
$$

Therefore, when $a$ and $b$ are almost equal, $G_{2}$ has the maximal Hosoya index.

Let $\mathcal{U}_{4}$ be the set of unicyclic graphs of the form $G_{4}$ (as depicted in Figure 1 ), where $v_{1}$ and $v_{2}$ are two vertices with $a-1, b-1$ pendent vertices, respectively, $a+b=n-3, a, b \geq 1$. Let $G_{4}^{*}$ be the graph of the form $G_{4}$ satisfying $|a-b| \leq 1$.

Theorem 4. The graph $G_{4}^{*}$ has the maximum Hosoya index among all graphs in $\mathcal{U}_{4}$. 
Proof. Assume $G_{4} \in \mathcal{U}_{4}$ with $a \geq 2, b \geq 1$. By Lemmas 1 and 2, we obtain

$$
\begin{aligned}
z\left(G_{4}\right) & =z\left(G_{4}-v_{1}\right)+\sum_{v^{\prime} \in N_{G_{4}}\left(v_{1}\right)} z\left(G_{4}-\left\{v_{1}, v^{\prime}\right\}\right) \\
& =z\left(G_{4}-v_{1}-v_{2}\right)+\sum_{y \in N_{G_{4}-v_{1}}\left(v_{2}\right)} z\left(G_{4}-v_{1}-\left\{v_{2}, y\right\}\right) \\
& +\sum_{v^{\prime} \in N_{G_{4}}\left(v_{1}\right)} z\left(G_{4}-\left\{v_{1}, v^{\prime}\right\}\right) \\
& =z\left(G_{4}-v_{1}-v_{2}\right)+\left[z\left(G_{4}-v_{1}-\left\{v_{2}, v_{3}\right\}\right)\right. \\
& \left.+(b-1) z\left(G_{4}-v_{1}-\left\{v_{2}, y\right\}\right)\right] \\
& +\left[z\left(G_{4}-\left\{v_{1}, v_{2}\right\}\right)+z\left(G_{4}-\left\{v_{1}, v_{5}\right\}\right)\right. \\
& \left.+(a-1) z\left(G_{4}-\left\{v_{1}, v^{\prime}\right\}\right)\right] \\
& =[3+2+3(b-1)]+[3+2 b+1+(a-1)(3 b+2)] \\
& =3 a b+2 a+2 b+4:=f_{4}(a, b),
\end{aligned}
$$

where $v^{\prime}$ is a pendent vertex adjacent to $v_{1}, y$ is a pendent vertex adjacent to $v_{2}$.

If $a-b \geq 2$, then we get

$$
f_{4}(a-1, b+1)-f_{4}(a, b)=3(a-b-1)>0 .
$$

This implies the result.

Theorem 5. The graph $G_{1}^{*}$ has the maximum Hosoya index among all graphs in $\mathcal{U}_{n}^{3}$ if $n \geq 17$.

Proof. We only need to compare the Hosoya indices of $G_{i}^{*}$ for $i=1,2,3,4$.

For $G_{1}^{*}$, we assume that $a \leq b \leq c$. As $a, b, c$ are almost equal and $a+b+c=n$, then we have $a \geq \frac{n-2}{3}$. Thus,

$$
\begin{aligned}
z\left(G_{1}^{*}\right) & =a b c+a+b+c \\
& \geq \frac{(n-2)^{3}}{27}+n:=g_{1}(n) .
\end{aligned}
$$

For $G_{2}^{*}$, as $0 \leq a-b \leq 2$ and $a+b=n-2$, we have $a-2 \leq b \leq a$ and thus $a \leq \frac{n}{2}$. Thus

$$
\begin{aligned}
z\left(G_{2}^{*}\right) & =2 a b+2 a+2 \\
& \leq 2 a^{2}+2 a+2 \\
& \leq \frac{n^{2}}{2}+n+2:=g_{2}(n) .
\end{aligned}
$$

The last inequality holds for a function $f(a)=2 a^{2}+2 a+2$ that is strictly increasing for $1 \leq a \leq \frac{n}{2}$. For $G_{3}^{*}$, as $a, b$ are almost equal and $a+b=n-2$, then we have

$$
\begin{aligned}
z\left(G_{3}^{*}\right) & =2 a b+a+b+3 \\
& \leq \frac{(a+b)^{2}}{2}+a+b+3 \\
& =\frac{(n-2)^{2}}{2}+n+1:=g_{3}(n) .
\end{aligned}
$$


For $G_{4}^{*}$, as $a, b$ are almost equal and $a+b=n-3$, then we have

$$
\begin{aligned}
z\left(G_{4}^{*}\right) & =3 a b+2 a+2 b+4 \\
& \leq \frac{3(a+b)^{2}}{4}+2(a+b)+4 \\
& =\frac{3(n-3)^{2}}{4}+2 n-2:=g_{4}(n) .
\end{aligned}
$$

By using the software "Mathematica", we see $g_{4}(n)>g_{2}(n)>g_{3}(n)$ for $n \geq 14, g_{1}(n)>g_{4}(n)$ for $n \geq 17$. A direct computation yields to $z\left(C_{5}\right)=11, z\left(C_{6}\right)=18, z\left(C_{7}\right)=29$.

From above, we obtain the result.

\section{The Unicyclic Graphs with Diameter 4}

In this section, we aim to determine the maximal Hosoya index of unicyclic graphs with $n$ vertices and diameter 4 .

Let $\mathcal{V}_{n}^{4}$ be the set of all unicyclic graphs with $n$ vertices and diameter 4 . According to the length of the unique cycle and the distribution of other vertices, we may classify all the members in $\mathcal{V}_{n}^{4}$. Let $\mathcal{V}_{i}$ be the set of unicyclic graphs of the form $H_{i}, i=1,2,3,4,5,6,7,8$. It is easy to see that the graphs from $\mathcal{V}_{1}, \mathcal{V}_{2}, \mathcal{V}_{3}, \mathcal{V}_{4}, \mathcal{V}_{5}, \mathcal{V}_{6}, \mathcal{V}_{7}, \mathcal{V}_{8}$, and $\mathcal{V}_{9}$ and two cycles $C_{8}$ and $C_{9}$ are all members of the unicyclic graphs with diameter 4 .

Let $\mathcal{V}_{1}$ be the set of unicyclic graphs of the form $H_{1}$ (as depicted in Figure 2), where $v_{1}, v_{4}$, and $v_{5}$ are three vertices with $c-1, b-1$, and $a-1$ pendent vertices, $a+b+c=n-2, a \geq 2, b \geq 1, c \geq 1$. Let $H_{1}^{*}$ be the graph of the form $H_{1}$ satisfying $0 \leq a-b \leq 1,0 \leq a-c \leq 2,0 \leq b-c \leq 1$.
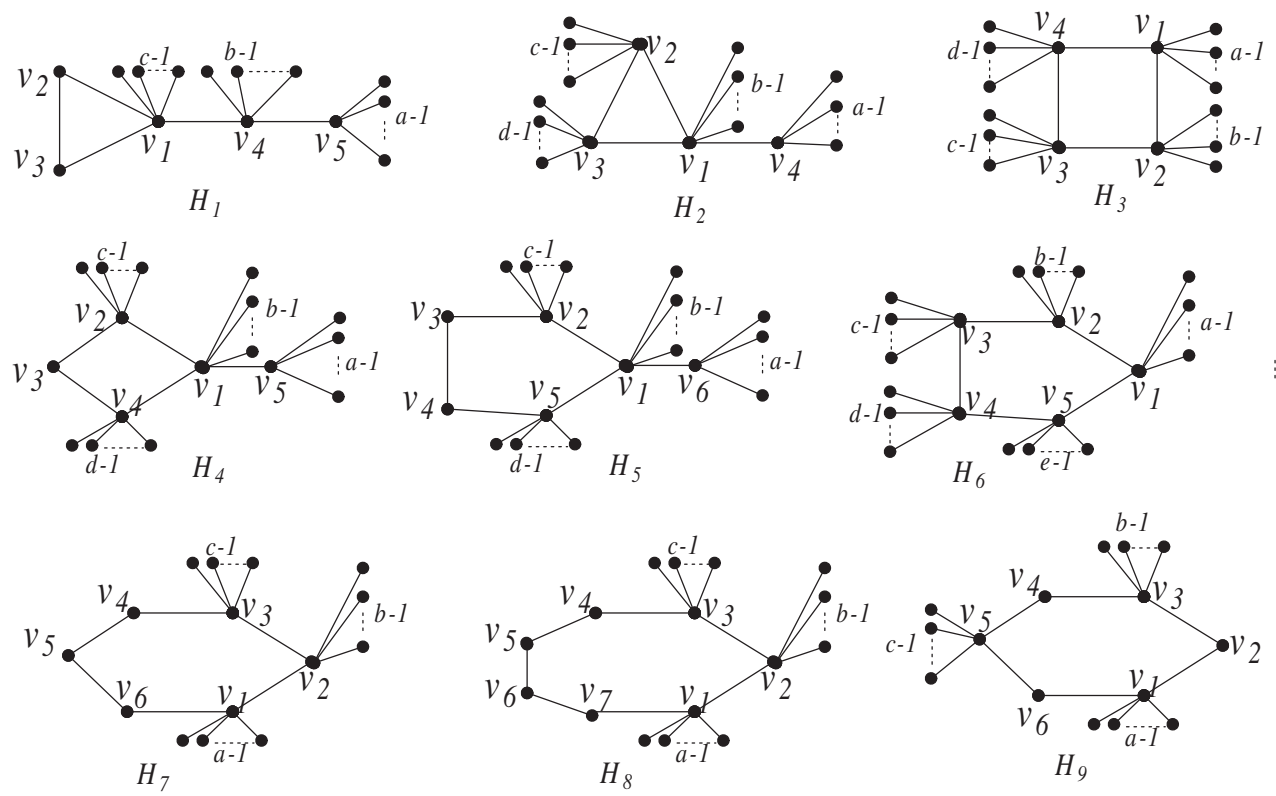

Figure 2. Eight unicyclic graphs with diameter 4.

Theorem 6. The graph $H_{1}^{*}$ has the maximum Hosoya index among all graphs in $\mathcal{V}_{1}$. 
Proof. For $H_{1} \in \mathcal{V}_{1}$, by Lemmas 1 and 2, we have

$$
\begin{aligned}
z\left(H_{1}\right) & =z\left(H_{1}-v_{1}\right)+\sum_{v^{\prime} \in N_{H_{1}}\left(v_{1}\right)} z\left(H_{1}-\left\{v_{1}, v^{\prime}\right\}\right) \\
& =z\left(H_{1}-v_{1}\right)+z\left(H_{1}-\left\{v_{1}, v_{2}\right\}\right)+z\left(H_{1}-\left\{v_{1}, v_{3}\right\}\right) \\
& +z\left(H_{1}-\left\{v_{1}, v_{4}\right\}\right)+(c-1) z\left(H_{1}-\left\{v_{1}, y\right\}\right) \\
& =2(a b+1)+(a b+1)+(a b+1)+2 a+2(c-1)(a b+1) \\
& =2 a b c+2 a b+2 a+2 c+2:=g_{1}(a, b, c),
\end{aligned}
$$

where $y$ is one pendent vertex adjacent to $v_{1}$ in $H_{1}$.

Case 1. If $b-a \geq 1$, then

$$
g_{1}(a+1, b-1, c)-g_{1}(a, b, c)=(b-a-1)(2 c+2)+2>0 .
$$

Case 2. If $a-b \geq 2$, then

$$
g_{1}(a-1, b+1, c)-g_{1}(a, b, c)=(a-b-1)(2 c+2)-2 \geq 2 c>0 .
$$

Cases 1 and 2 imply that, if $H_{1}$ has maximum Hosoya index, then we infer $0 \leq a-b \leq 1$.

Case 3. If $c-a \geq 1$, then

$$
g_{1}(a+1, b, c-1)-g_{1}(a, b, c)=2 b(c-a-1)+2 b>0 .
$$

Case 4. If $a-c \geq 3$, then

$$
g_{1}(a-1, b, c+1)-g_{1}(a, b, c)=2 b(a-c-2) \geq 2 b>0 .
$$

Cases 3 and 4 imply that, if $H_{1}$ has maximum Hosoya index, then we infer $0 \leq a-c \leq 2$.

Case 5. If $c-b \geq 1$, then

$$
g_{1}(a, b+1, c-1)-g_{1}(a, b, c)=2 a(c-b)-2>0 .
$$

Case 6. If $b-c \geq 2$, then

$$
g_{1}(a, b-1, c+1)-g_{1}(a, b, c)=2 a(b-c-2)+2>0 .
$$

Cases 5 and 6 imply that if $H_{1}$ has maximum Hosoya index, then we infer $0 \leq b-c \leq 1$ are almost equal.

From the above, we get the result.

Let $\mathcal{V}_{2}$ be the set of unicyclic graphs of the form $H_{2}$ (as depicted in Figure 2), where $v_{4}, v_{1}, v_{2}$, and $v_{3}$ are four vertices with $a-1, b-1, c-1$, and $d-1$ pendent vertices, respectively, $a+b+c+d=n$. As the diameter is 4 , we infer $a \geq 2$ and one value of $c$ and $d$ is at least 2 . Let $H_{2}^{*}$ be the graph of the form $H_{2}$ satisfying $0 \leq a-b \leq 2,0 \leq a-c \leq 1,|c-d| \leq 1$.

Theorem 7. The graph $H_{2}^{*}$ has the maximum Hosoya index among all graphs in $\mathcal{V}_{2}$. 
Proof. For $\mathrm{H}_{2} \in \mathcal{V}_{2}$, by Lemmas 1 and 2, we have

$$
\begin{aligned}
z\left(H_{2}\right) & =z\left(H_{2}-v_{1}\right)+\sum_{v^{\prime} \in N_{H_{2}}\left(v_{1}\right)} z\left(H_{2}-\left\{v_{1}, v^{\prime}\right\}\right) \\
& =z\left(H_{2}-v_{1}\right)+z\left(H_{2}-\left\{v_{1}, v_{2}\right\}\right)+z\left(H_{2}-\left\{v_{1}, v_{3}\right\}\right) \\
& +z\left(H_{2}-\left\{v_{1}, v_{4}\right\}\right)+(b-1) z\left(H_{2}-\left\{v_{1}, y\right\}\right) \\
& =a(c d+1)+a d+a c+(c d+1)+a(b-1)(c d+1) \\
& =a b c d+a b+a c+a d+c d+1:=g_{2}(a, b, c, d)
\end{aligned}
$$

where $y$ is one of pendent vertex adjacent to $v_{1}$ in $H_{2}$.

Case 1. If $c-d \geq 2$, then

$$
g_{2}(a, b, c-1, d+1)-g_{2}(a, b, c, d)=(a b+1)(c-d-1)>0 .
$$

Case 2. If $d-c \geq 2$, we infer

$$
g_{2}(a, b, c+1, d-1)-g_{2}(a, b, c, d)=(a b+1)(d-c-1)>0 .
$$

Cases 1 and 2 imply that, if $H_{2}$ has maximum Hosoya index, then we infer $|c-d| \leq 1$.

Case 3. If $a-b \geq 3$, then

$$
\begin{aligned}
g_{2}(a-1, b+1, c, d)-g_{2}(a, b, c, d) & =(a-b-1) c d \\
& +(a-b-1)-c-d \\
& \geq 2 c d+2-c-d \\
& =c d+1+(c-1)(d-1)>0 .
\end{aligned}
$$

Case 4. If $b-a \geq 1$, then

$$
\begin{aligned}
g_{2}(a+1, b-1, c, d)-g_{2}(a, b, c, d) & =(b-a-1) c d \\
& +(b-a-1)+c+d \\
& \geq c+d>0 .
\end{aligned}
$$

Cases 3 and 4 imply that, if $H_{2}$ has maximum Hosoya index, then infer $0 \leq a-b \leq 2$.

Case 5. If $a-c \geq 2$, then

$$
\begin{aligned}
g_{2}(a-1, b, c+1, d)-g_{2}(a, b, c, d) & =(a-c-1) b d \\
& -b+(a-c-1) \\
& \geq b d-b+1>0 .
\end{aligned}
$$

Case 6. If $c-a \geq 1$, then

$$
\begin{aligned}
g_{2}(a+1, b, c-1, d)-g_{2}(a, b, c, d) & =(c-a-1) b d \\
& +b+(c-a-1) \\
& \geq b>0 .
\end{aligned}
$$

Cases 5 and 6 imply that, if $H_{2}$ has maximum Hosoya index, then we infer $0 \leq a-c \leq 1$.

From the above cases, we get the result.

Let $\mathcal{V}_{3}$ be the set of unicyclic graphs of the form $H_{3}$ (as depicted in Figure 2), where $v_{1}, v_{2}, v_{3}$, and $v_{4}$ are four vertices with $a-1, b-1, c-1$, and $d-1$ pendent vertices, $a+b+c+d=n, a, b, c, d \geq 1$. 
As the diameter is $4, a$ and $c$, or $b$ and $d$, are at least 2. Let $H_{3}^{*}$ be the graph of the form $H_{3}$ satisfying $a, b, c, d$ almost equal.

Theorem 8. The graph $H_{3}^{*}$ has the maximum Hosoya index among all graphs in $\mathcal{V}_{3}$.

Proof. For $\mathrm{H}_{3} \in \mathcal{V}_{3}$, by Lemmas 1 and 2, we have

$$
z\left(H_{3}\right)=z\left(H_{3}-v_{1}\right)+\sum_{v^{\prime} \in N_{H_{3}}\left(v_{1}\right)} z\left(H_{3}-\left\{v_{1}, v^{\prime}\right\}\right) .
$$

For $z\left(H_{3}-v_{1}\right)$, by Lemma 1 , we have

$$
\begin{aligned}
z\left(H_{3}-v_{1}\right) & =z\left(H_{3}-v_{1}-v_{2}\right)+\sum_{v^{\prime} \in N_{H_{3}-v_{1}}\left(v_{2}\right)} z\left(H_{3}-v_{1}-\left\{v_{2}, v^{\prime}\right\}\right) \\
& =z\left(H_{3}-v_{1}-v_{2}\right) \\
& +z\left(H_{3}-v_{1}-\left\{v_{2}, v_{3}\right\}\right) \\
& +(b-1) z\left(H_{3}-v_{1}-\left\{v_{2}, y\right\}\right) \\
& =(c d+1)+d+(b-1)(c d+1) \\
& =b c d+b+d,
\end{aligned}
$$

where $y$ is one pendent vertex adjacent to $v_{2}$ in $H_{3}$.

For $\sum_{v^{\prime} \in N_{H_{3}}}\left(v_{1}\right) z\left(H_{3}-\left\{v_{1}, v^{\prime}\right\}\right)$, by Lemmas 1 and 2, we have

$$
\begin{aligned}
& \sum_{v^{\prime} \in N_{H_{3}}\left(v_{1}\right)} z\left(H_{3}-\left\{v_{1}, v^{\prime}\right\}\right) \\
= & z\left(H_{3}-\left\{v_{1}, v_{2}\right\}\right)+z\left(H_{3}-\left\{v_{1}, v_{4}\right\}\right)+(a-1) z\left(H_{3}-\left\{v_{1}, w\right\}\right) \\
= & (c d+1)+(b c+1)+(a-1)(b c d+b+d)
\end{aligned}
$$

where $w$ is one of pendent vertex adjacent to $v_{1}$ in $H_{3}$. Therefore,

$$
\begin{aligned}
z\left(H_{3}\right) & =z\left(H_{3}-v_{1}\right)+\sum_{v^{\prime} \in N_{H_{3}}\left(v_{1}\right)} z\left(H_{3}-\left\{v_{1}, v^{\prime}\right\}\right) \\
& =(b c d+b+d)+[(c d+1)+(b c+1)+(a-1)(b c d+b+d)] \\
& =a b c d+a b+a d+b c+c d+2:=g_{3}(a, b, c, d) .
\end{aligned}
$$

Case 1. If $a-c \geq 2$, then

$$
g_{3}(a-1, b, c+1, d)-g_{3}(a, b, c, d)=b d(a-c-1)>0 .
$$

From Case 1, we have, when $a$ and $c$ are almost equal, $H_{3}$ has larger Hosoya index. Similarly, as $b, d$ have the same status as shown in the graph, we conclude that, when $b$ and $d$ are almost equal, $\mathrm{H}_{3}$ has larger Hosoya index.

Case 2. If $a-b \geq 2$, then

$$
\begin{aligned}
g_{3}(a-1, b+1, c, d)-g_{3}(a, b, c, d) & =(a-b-1) c d \\
& +(a-b-1)-d+c \\
& \geq c d+1-d+c \\
& =(c-1)(d+1)+2>0 .
\end{aligned}
$$


Therefore, from Case 2, when $\mathrm{H}_{3}$ has maximum Hosoya index, we infer that $a$ and $b$ are almost equal, and, similarly, $a$ and $d$ are almost equal.

From Case $1, b$ and $d$ are almost equal, so $a, b$ and $d$ are almost equal. Similarly, $a, b$ and $c$ are almost equal. Hence, we get $a, b, c, d$ are almost equal.

Let $\mathcal{V}_{4}$ be the set of unicyclic graphs of the form $H_{4}$ (as depicted in Figure 2), where $v_{5}, v_{1}, v_{2}$, and $v_{4}$ are four vertices with $a-1, b-1, c-1$, and $d-1$ pendent vertices with $a+b+c+d=n$, $a \geq 2, b, c, d \geq 1$. Let $H_{4}^{*}$ be the graph of the form $H_{4}$ satisfying $0 \leq a-b \leq 2,|c-d| \leq 1,0 \leq a-c \leq 1$.

Theorem 9. The graph $H_{4}^{*}$ has the maximum Hosoya index among all graphs in $\mathcal{V}_{4}$.

Proof. For $H_{4} \in \mathcal{V}_{4}$, by Lemmas 1 and 2, we have

$$
z\left(H_{4}\right)=z\left(H_{4}-v_{1}\right)+\sum_{v^{\prime} \in N_{H_{4}}\left(v_{1}\right)} z\left(H_{4}-\left\{v_{1}, v^{\prime}\right\}\right)
$$

For $z\left(H_{4}-v_{1}\right)$, by Lemma 1 , we get

$$
\begin{aligned}
z\left(H_{4}-v_{1}\right) & =z\left(H_{4}-v_{1}-v_{2}\right)+\sum_{v^{\prime} \in N_{H_{4}-v_{1}}\left(v_{2}\right)} z\left(H_{4}-v_{1}-\left\{v_{2}, v^{\prime}\right\}\right) \\
& =z\left(H_{4}-v_{1}-v_{2}\right)+z\left(H_{4}-v_{1}-\left\{v_{2}, v_{3}\right\}\right) \\
& +(c-1) z\left(H_{4}-v_{1}-\left\{v_{2}, y\right\}\right) \\
& =a(d+1)+a d+(c-1) a(d+1) \\
& =a c d+a c+a d
\end{aligned}
$$

where $y$ is one pendent vertex adjacent to $v_{2}$ in $H_{4}$. For $\sum_{v^{\prime} \in N_{H_{4}}\left(v_{1}\right)} z\left(H_{4}-\left\{v_{1}, v^{\prime}\right\}\right)$, by Lemmas 1 and 2,

$$
\begin{aligned}
\sum_{v^{\prime} \in N_{H_{4}}\left(v_{1}\right)} z\left(H_{4}-\left\{v_{1}, v^{\prime}\right\}\right) & =z\left(H_{4}-\left\{v_{1}, v_{2}\right\}\right)+z\left(H_{4}-\left\{v_{1}, v_{4}\right\}\right) \\
& +z\left(H_{4}-\left\{v_{1}, v_{5}\right\}\right) \\
& +(b-1) z\left(H_{4}-\left\{v_{1}, v_{b-1}\right\}\right) \\
& =z\left(H_{4}-\left\{v_{1}, v_{2}\right\}\right) \\
& +z\left(H_{4}-\left\{v_{1}, v_{4}\right\}\right) \\
& +\left[z\left(H_{4}-\left\{v_{1}, v_{5}\right\}-v_{2}\right)\right. \\
& +z\left(H_{4}-\left\{v_{1}, v_{5}\right\}-\left\{v_{2}, v_{3}\right\}\right) \\
& \left.+(c-1) z\left(H_{4}-\left\{v_{1}, v_{5}\right\}-\left\{v_{2}, y\right\}\right)\right] \\
& +(b-1) z\left(H_{4}-\left\{v_{1}, w\right\}\right) \\
& =a(d+1)+a(c+1) \\
& +(c d+c+d) \\
& +(b-1)(a c d+a c+a d)
\end{aligned}
$$

where $w$ is one pendent vertex adjacent to $v_{1}$ in $H_{4}$. Hence, it follows that 


$$
\begin{aligned}
z\left(H_{4}\right) & =z\left(H_{4}-v_{1}\right)+\sum_{v^{\prime} \in N_{H_{4}}\left(v_{1}\right)} z\left(H_{4}-\left\{v_{1}, v^{\prime}\right\}\right) \\
& =(a c d+a c+a d) \\
& +[a(d+1)+a(c+1) \\
& +(c d+c+d)+(b-1)(a c d+a c+a d)] \\
& =a b c d+a b c+a b d+a c+a d+c d+2 a+c+d \\
& :=g_{4}(a, b, c, d) .
\end{aligned}
$$

Case 1. If $c-d \geq 2$, then

$$
\begin{aligned}
g_{4}(a, b, c-1, d+1)-g_{4}(a, b, c, d) & -(a b c d+a b c \\
& +a b d+a c+a d \\
& +c d+2 a+c+d) \\
& =(a b+1)(c-d-1)>0 .
\end{aligned}
$$

Similarly, if $d-c \geq 2$, then we have

$$
g_{4}(a, b, c+1, d-1)-g_{4}(a, b, c, d)=(a b+1)(d-c-1)>0 .
$$

Cases 1 implies that, if $H_{4}$ has the maximum Hosoya index, we conclude $|d-c| \leq 1$.

Case 2. If $a-b \geq 3$, then

$$
\begin{aligned}
g_{4}(a-1, b+1, c, d)-g_{4}(a, b, c, d) & =(a-b-1) c d \\
& +(a-b-1)(c+d) \\
& -c-d-2 \\
& \geq 2 c d+c+d-2>0 .
\end{aligned}
$$

Case 3. If $b-a \geq 1$, then

$$
\begin{aligned}
& g_{4}(a+1, b-1, c, d)-g_{4}(a, b, c, d) \\
& =(b-a-1) c d+(b-a-1)(c+d)+c+d+2 \\
& \geq c+d+2>0 .
\end{aligned}
$$

Cases 2 and 3 imply that, if $H_{4}$ has the maximum Hosoya index, we conclude $0 \leq a-b \leq 2$.

Case 4. If $a-c \geq 2$, then

$$
\begin{aligned}
g_{4}(a-1, b, c+1, d)-g_{4}(a, b, c, d) & =(a-c-1) b d \\
& +(a-c-1) b \\
& -b d+(a-c-1)-1>0 .
\end{aligned}
$$

Case 5. If $c-a \geq 1$, then

$$
\begin{aligned}
g_{4}(a+1, b, c-1, d)-g_{4}(a, b, c, d) & =(c-a-1) b d \\
& +(c-a-1) b+b d \\
& +(c-a-1)+1>0 .
\end{aligned}
$$

Thus, we have $z\left(H_{46}\right)>z\left(H_{4}\right)$.

Cases 4 and 5 imply that, if $H_{4}$ has the maximum Hosoya index, we conclude $0 \leq a-c \leq 1$.

All the above cases imply the desired result. 
Let $\mathcal{V}_{5}$ be the set of unicyclic graphs of the form $H_{5}$ (as depicted in Figure 2), where $v_{6}, v_{1}, v_{2}$, and $v_{5}$ are four vertices with $a-1, b-1, c-1$, and $d-1$ pendent vertices, $a+b+c+d=n-2$, and $a \geq$ $2, b, c, d \geq 1$. Let $H_{5}^{*}$ be the graph of the form $H_{5}$ satisfying $0 \leq a-b \leq 2,|c-d| \leq 1,0 \leq a-c \leq 1$.

Theorem 10. The graph $H_{5}^{*}$ has the maximum Hosoya index among all graphs in $\mathcal{V}_{5}$.

Proof. For $\mathrm{H}_{5} \in \mathcal{V}_{5}$, by Lemmas 1 and 2,

$$
\begin{aligned}
z\left(H_{5}\right) & =z\left(H_{5}-v_{1}\right)+\sum_{v^{\prime} \in N_{H_{5}}\left(v_{1}\right)} z\left(H_{5}-\left\{v_{1}, v^{\prime}\right\}\right) \\
& =z\left(H_{5}-v_{1}\right)+z\left(H_{5}-\left\{v_{1}, v_{2}\right\}\right)+z\left(H_{5}-\left\{v_{1}, v_{5}\right\}\right) \\
& +z\left(H_{5}-\left\{v_{1}, v_{6}\right\}\right)+(b-1) z\left(H_{5}-\left\{v_{1}, y\right\}\right)
\end{aligned}
$$

where $y$ is one pendent vertex adjacent to $v_{1}$ in $H_{5}$.

For $z\left(H_{5}-v_{1}\right)$, By Lemma 1 , we have

$$
\begin{aligned}
z\left(H_{5}-v_{1}\right) & =z\left(H_{5}-v_{1}-v_{2}\right)+\sum_{v^{\prime} \in N_{H_{5}-v_{1}}\left(v_{2}\right)} z\left(H_{5}-v_{1}-\left\{v_{2}, v^{\prime}\right\}\right) \\
& =z\left(H_{5}-v_{1}-v_{2}\right)+z\left(H_{5}-v_{1}-\left\{v_{2}, v_{3}\right\}\right) \\
& +(c-1) z\left(H_{5}-v_{1}-\left\{v_{2}, w\right\}\right) \\
& =a(2 d+1)+a(d+1)+(c-1) a(2 d+1) \\
& =2 a c d+a c+a d+a,
\end{aligned}
$$

where $w$ is one pendent vertex adjacent to $v_{2}$ in $H_{5}, z\left(H_{5}-\left\{v_{1}, v_{2}\right\}\right)=a(2 d+1)$, and $z\left(H_{5}-\right.$ $\left.\left\{v_{1}, v_{3}\right\}\right)=a(2 c+1)$. Therefore,

$$
\begin{aligned}
z\left(H_{5}\right) & =(2 a c d+a c+a d+a)+a(2 d+1)+a(2 c+1) \\
& +(2 c d+c+d+1)+(b-1)(2 a c d+a c+a d+a) \\
& =2 a b c d+a b c+a b d+a b+2 a c \\
& +2 a d+2 c d+2 a+c+d+1:=g_{5}(a, b, c, d) .
\end{aligned}
$$

Case 1. If $c-d \geq 2$, then

$$
g_{5}(a, b, c-1, d+1)-g_{5}(a, b, c, d)=(2 a b+2)(c-d-1)>0 .
$$

Case 2. If $d-c \geq 2$, this yields to

$$
g_{5}(a, b, c+1, d-1)-g_{5}(a, b, c, d)=(2 a b+2)(d-c-1)>0 .
$$

Cases 1 and 2 imply that, if $H_{5}$ has the maximum Hosoya index, we infer $|d-c| \leq 1$.

Case 3. If $a-b \geq 3$, then

$$
\begin{aligned}
g_{5}(a-1, b+1, c, d)-g_{5}(a, b, c, d) & =(2 c d+c+d+1)(a-b-1) \\
& -2 c-2 d-2 \\
& \geq 4 c d>0 .
\end{aligned}
$$

Case 4. If $b-a \geq 1$, then

$$
\begin{aligned}
g_{5}(a+1, b-1, c, d)-g_{5}(a, b, c, d) & =(2 c d+c+d+1)(b-a-1) \\
& +2 c+2 d+2>0 .
\end{aligned}
$$


Cases 3 and 4 imply that, if $H_{5}$ has the maximum Hosoya index, we conclude $0 \leq a-b \leq 2$.

Case 5. If $a-c \geq 2$, then

$$
\begin{aligned}
g_{5}(a-1, b, c+1, d)-g_{5}(a, b, c, d) & =(2 b d+b+2)(a-c-1) \\
& -b d-b-1 \geq b d+1>0 .
\end{aligned}
$$

Case 6. If $c-a \geq 1$, then

$$
\begin{aligned}
g_{5}(a+1, b, c-1, d)-g_{5}(a, b, c, d) & =(2 b d+b+2)(c-a-1) \\
& +b d+b+1>0 .
\end{aligned}
$$

Cases 5 and 6 imply that, if $H_{5}$ has the maximum Hosoya index, we conclude $0 \leq a-c \leq 1$.

From all above, we get the result.

Let $\mathcal{V}_{6}$ be the set of unicyclic graphs of the form $H_{6}$ (as depicted in Figure 2), where $v_{1}, v_{2}, v_{3}, v_{4}$, and $v_{5}$ are five vertices with $a-1, b-1, c-1, d-1$, and $e-1$ pendent vertices, $a+b+c+d+e=n$, and $a, b, c, d, e \geq 1$. As the diameter is 4 , from symmetry, we may assume $a$ and $c$, or $a$ and $d$, are at least 2. Let $H_{6}^{*}$ be the graph of the form $H_{6}$ satisfying $a, b, c, d, e$ almost equal.

Theorem 11. The graph $H_{6}^{*}$ has the maximum Hosoya index among all graphs in $\mathcal{V}_{6}$.

Proof. For $H_{6} \in \mathcal{V}_{6}$, by Lemmas 1 and 2, we have

$$
\begin{aligned}
z\left(H_{6}\right) & =z\left(H_{6}-v_{1}\right)+\sum_{v^{\prime} \in N_{H_{6}}\left(v_{1}\right)} z\left(H_{6}-\left\{v_{1}, v^{\prime}\right\}\right) \\
& =z\left(H_{6}-v_{1}\right)+z\left(H_{6}-\left\{v_{1}, v_{2}\right\}\right)+z\left(H_{6}-\left\{v_{1}, v_{5}\right\}\right) \\
& +(a-1) z\left(H_{6}-\left\{v_{1}, y\right\}\right)
\end{aligned}
$$

where $y$ is one pendent vertex adjacent to $v_{1}$ in $H_{6}$.

For $z\left(H_{6}-v_{1}\right)$, by Lemma 1 , we have

$$
\begin{aligned}
z\left(H_{6}-v_{1}\right) & =z\left(H_{6}-v_{1}-v_{2}\right)+\sum_{v^{\prime} \in N_{H_{6}-v_{1}}\left(v_{2}\right)} z\left(H_{6}-v_{1}-\left\{v_{2}, v^{\prime}\right\}\right) \\
& =z\left(H_{6}-v_{1}-v_{2}\right)+z\left(H_{6}-v_{1}-\left\{v_{2}, v_{3}\right\}\right) \\
& +(b-1) z\left(H_{6}-v_{1}-\left\{v_{2}, v_{b-1}\right\}\right) \\
& =\left[z\left(H_{6}-v_{1}-v_{2}-v_{3}\right)+z\left(H_{6}-v_{1}-v_{2}-\left\{v_{3}, v_{4}\right\}\right)\right. \\
& \left.+(c-1) z\left(H_{6}-v_{1}-v_{2}-\left\{v_{3}, v_{c-1}\right\}\right)\right] \\
& +z\left(H_{6}-v_{1}-\left\{v_{2}, v_{3}\right\}\right) \\
& +(b-1) z\left(H_{6}-v_{1}-\left\{v_{2}, v_{b-1}\right\}\right) \\
& =[(d e+1)+e+(c-1)(d e+1)] \\
& +(d e+1)+(b-1)(c d e+c+e) \\
& =b c d e+b c+b e+d e+1,
\end{aligned}
$$

where $v_{b-1}$ is one pendent vertex adjacent to $v_{2}$ in $H_{6}, v_{c-1}$ is one pendent vertex adjacent to $v_{3}$, 
$z\left(H_{6}-\left\{v_{1}, v_{2}\right\}\right)=c d e+c+e$, and

$$
\begin{aligned}
z\left(H_{6}-\left\{v_{1}, v_{5}\right\}\right) & =z\left(H_{6}-\left\{v_{1}, v_{5}\right\}-v_{4}\right)+z\left(H_{6}-\left\{v_{1}, v_{5}\right\}\right. \\
& \left.-\left\{v_{4}, v_{3}\right\}\right)+(d-1) z\left(H_{6}-\left\{v_{1}, v_{5}\right\}\right. \\
& \left.-\left\{v_{4}, v_{d-1}\right\}\right) \\
& =(b c+1)+b+(d-1)(b c+1) \\
& =b c d+b+d .
\end{aligned}
$$

Therefore, we obtain

$$
\begin{aligned}
z\left(H_{6}\right) & =(b c d e+b c+b e+d e+1) \\
& +(c d e+c+e)+(b c d+b+d) \\
& +(a-1)(b c d e+b c+b e+d e+1) \\
& =a b c d e+a b c+a b e+a d e \\
& +b c d+c d e+a+b+c+d+e .
\end{aligned}
$$

If $a-b \geq 2$, let $H_{6}^{\prime}$ be the graph obtained from $H_{6}$ by removing a pendent edge at $v_{1}$ to $v_{2}$. Then, we get

$$
\begin{aligned}
z\left(H_{6}^{\prime}\right)-z\left(H_{6}\right) & =(a-b-1) c d e+(a-b-1)(c+e)-d e+c d \\
& \geq c d e+c+e-d e+c d \\
& =(c-1) d e+c d+c+e>0 .
\end{aligned}
$$

As $a, b, c, d, e$ have the same status as depicted in Figure 2, we obtain that, when $a, b, c, d, e$ are almost equal, $H_{6}$ has the maximal Hosoya index.

Let $\mathcal{V}_{7}$ be the set of unicyclic graphs of the form $H_{7}$ (as depicted in Figure 2), where $v_{1}, v_{2}$, and $v_{3}$ are three vertices with $a-1, b-1$, and $c-1$ pendent vertices, $a+b+c=n-3, a, b, c \geq 1$, and one of $a, b, c$ is at least 2 . Let $H_{7}^{*}$ be the graph of the form $H_{7}$ satisfying $a, b, c$ almost equal.

Theorem 12. The graph $H_{7}^{*}$ has the maximum Hosoya index among all graphs in $\mathcal{V}_{7}$.

Proof. For $H_{7} \in \mathcal{V}_{7}$, by Lemmas 1 and 2, we have

$$
\begin{aligned}
z\left(H_{7}\right) & =z\left(H_{7}-v_{1}\right)+\sum_{v^{\prime} \in N_{H_{7}}\left(v_{1}\right)} z\left(H_{7}-\left\{v_{1}, v^{\prime}\right\}\right) \\
& =z\left(H_{7}-v_{1}\right)+z\left(H_{7}-\left\{v_{1}, v_{2}\right\}\right) \\
& +z\left(H_{7}-\left\{v_{1}, v_{6}\right\}\right)+(a-1) z\left(H_{7}-\left\{v_{1}, v_{a-1}\right\}\right),
\end{aligned}
$$

where $v_{a-1}$ is one pendent vertex adjacent to $v_{1}$ in $H_{7}$.

For $z\left(H_{7}-v_{1}\right)$, by Lemma 1 , we have

$$
\begin{aligned}
z\left(H_{7}-v_{1}\right) & =z\left(H_{7}-v_{1}-v_{2}\right)+\sum_{v^{\prime} \in N_{H_{7}-v_{1}}\left(v_{2}\right)} z\left(H_{7}-v_{1}-\left\{v_{2}, v^{\prime}\right\}\right) \\
& =[3+2+3(c-1)]+3+(b-1)(3 c+2) \\
& =3 b c+2 b+3,
\end{aligned}
$$

where $v_{c-1}$ is one pendent vertex adjacent to $v_{3}, v_{b-1}$ is one pendent vertex adjacent to $v_{2}$ in $H_{7}$, 
$z\left(H_{7}-\left\{v_{1}, v_{2}\right\}\right)=3 c+2$, and

$$
\begin{aligned}
z\left(H_{7}-\left\{v_{1}, v_{6}\right\}\right) & =[b(c+1)+1]+b(c+1) \\
& =2 b c+b+2
\end{aligned}
$$

Therefore, we obtain

$$
\begin{aligned}
z\left(H_{7}\right) & =(3 b c+2 b+3)+(3 c+2) \\
& +(2 b c+b+2)+(a-1)(3 b c+2 b+3) \\
& =3 a b c+2 a b+2 b c+3 a+b+3 c+4:=g_{7}(a, b, c) .
\end{aligned}
$$

Case 1. If $a-b \geq 2$, then

$$
\begin{aligned}
g_{7}(a-1, b+1, c)-g_{7}(a, b, c) & =(3 c+2)(a-b-1) \\
& +2 c-2 \geq 5 c>0 .
\end{aligned}
$$

Case 2. If $b-a \geq 2$, then we conclude

$$
\begin{aligned}
g_{7}(a+1, b-1, c)-g_{7}(a, b, c) & =(3 c+2)(b-a-1) \\
& -2 c+2>0 .
\end{aligned}
$$

Cases 1 and 2 imply that, if $H_{7}$ has the maximum Hosoya index, we conclude $|a-b| \leq 1$. Similarly, by using symmetry, we may obtain $|c-b| \leq 1$.

Case 3. If $a-c \geq 2$, then

$$
g_{7}(a-1, b, c+1)-g_{7}(a, b, c)=3 b(a-c-1)>0 .
$$

Case 4. If $c-a \geq 2$, then get

$$
g_{7}(a+1, b, c-1)-g_{7}(a, b, c)=3 b(c-a-1)>0 .
$$

Cases 3 and 4 imply that, if $H_{7}$ has the maximum Hosoya index, we conclude $|a-c| \leq 1$.

In conclusion, when $a, b, c$ are almost equal, $H_{7}$ has the maximal Hosoya index.

Let $\mathcal{V}_{8}$ be the set of unicyclic graphs of the form $H_{8}$ (as depicted in Figure 2), where $v_{1}, v_{2}$, and $v_{3}$ are three vertices with $a-1, b-1$, and $c-1$ pendent vertices, $a+b+c=n-4, a, b, c \geq 1$, NS one of $a, b, c$ is at least 2 . Let $H_{8}^{*}$ be the graph of the form $H_{8}$ satisfying $a, b, c$ almost equal.

Theorem 13. The graph $H_{8}^{*}$ has the maximum Hosoya index among all graphs in $\mathcal{V}_{8}$.

Proof. For $H_{8} \in \mathcal{V}_{8}$, by Lemmas 1 and 2, we have

$$
\begin{aligned}
z\left(H_{8}\right) & =z\left(H_{8}-v_{1}\right)+\sum_{v^{\prime} \in N_{H_{8}}\left(v_{1}\right)} z\left(H_{8}-\left\{v_{1}, v^{\prime}\right\}\right) \\
& =z\left(H_{8}-v_{1}\right)+z\left(H_{8}-\left\{v_{1}, v_{2}\right\}\right) \\
& +z\left(H_{8}-\left\{v_{1}, v_{7}\right\}\right)+(a-1) z\left(H_{8}-\left\{v_{1}, v_{1, a-1}\right\}\right),
\end{aligned}
$$

where $v_{a-1}$ is one pendent vertex adjacent to $v_{1}$ in $H_{8}$. 
For $z\left(H_{8}-v_{1}\right)$, by Lemma 1 , we have

$$
\begin{aligned}
z\left(H_{8}-v_{1}\right) & =z\left(H_{8}-v_{1}-v_{2}\right) \\
& +\sum_{v^{\prime} \in N_{H_{8}-v_{1}}\left(v_{2}\right)} z\left(H_{8}-v_{1}-\left\{v_{2}, v^{\prime}\right\}\right) \\
& =z\left(H_{8}-v_{1}-v_{2}\right)+z\left(H_{8}-v_{1}-\left\{v_{2}, v_{3}\right\}\right) \\
& +(b-1) z\left(H_{8}-v_{1}-\left\{v_{2}, v_{b-1}\right\}\right) \\
& =\left[z\left(H_{8}-v_{1}-v_{2}-v_{3}\right)\right. \\
& +z\left(H_{8}-v_{1}-v_{2}-\left\{v_{3}, v_{4}\right\}\right) \\
& \left.+(c-1) z\left(H_{8}-v_{1}-v_{2}-\left\{v_{3}, v_{c-1}\right\}\right)\right] \\
& +z\left(H_{8}-v_{1}-\left\{v_{2}, v_{3}\right\}\right) \\
& +(b-1) z\left(H_{8}-v_{1}-\left\{v_{2}, v_{b-1}\right\}\right) \\
& =[5+3+5(c-1)]+5+(b-1)(5 c+3) \\
& =5 b c+3 b+5,
\end{aligned}
$$

where $v_{c-1}$ is one pendent vertex adjacent to $v_{3}$ in $H_{8}, v_{b-1}$ is one pendent vertex adjacent to $v_{2}$ in $H_{8}$, $z\left(H_{8}-\left\{v_{1}, v_{2}\right\}\right)=5 c+3$, and

$$
\begin{aligned}
z\left(H_{8}-\left\{v_{1}, v_{7}\right\}\right) & =z\left(H_{8}-\left\{v_{1}, v_{7}\right\}-v_{6}\right) \\
& +z\left(H_{8}-\left\{v_{1}, v_{7}\right\}-\left\{v_{6}, v_{5}\right\}\right) \\
& =z\left(H_{8}-\left\{v_{1}, v_{7}\right\}-v_{6}-v_{5}\right) \\
& \left.+z\left(H_{8}-\left\{v_{1}, v_{7}\right\}-v_{6}-\left\{v_{5}, v_{4}\right\}\right)\right] \\
& +z\left(H_{8}-\left\{v_{1}, v_{7}\right\}-\left\{v_{6}, v_{5}\right\}\right) \\
& =2[b(c+1)+1]+b c+1 \\
& =3 b c+2 b+3 .
\end{aligned}
$$

Therefore,

$$
\begin{aligned}
z\left(H_{8}\right) & =(5 b c+3 b+5)+(5 c+3) \\
& +(3 b c+2 b+3)+(a-1)(5 b c+3 b+5) \\
& =5 a b c+3 a b+3 b c+5 a+2 b+5 c+6:=g_{8}(a, b, c) .
\end{aligned}
$$

Case 1. If $a-b \geq 2$, then

$$
\begin{aligned}
g_{8}(a-1, b+1, c)-g_{8}(a, b, c) & =(5 c+3)(a-b-1) \\
& +3 c-3 \geq 8 c>0 .
\end{aligned}
$$

Case 2. If $b-a \geq 2$, then

$$
\begin{aligned}
g_{8}(a+1, b-1, c)-g_{8}(a, b, c) & =(5 c+3)(b-a-1) \\
& -3 c+3 \geq 2 c+6>0 .
\end{aligned}
$$

Cases 1 and 2 imply that, if $H_{8}$ has the maximum Hosoya index, we conclude $|a-b| \leq 1$. Similarly, we obtain $|c-b| \leq 1$.

Case 3. If $a-c \geq 2$, then

$$
g_{8}(a-1, b, c+1)-g_{8}(a, b, c)=5 b(a-c-1)>0 .
$$


Case 4. If $c-a \geq 2$, then we have

$$
g_{8}(a+1, b, c-1)-g_{8}(a, b, c)=5 b(c-a-1)>0 .
$$

Cases 3 and 4 imply that, if $H_{8}$ has the maximum Hosoya index, we conclude $|a-c| \leq 1$.

In conclusion, when $a, b, c$ are almost equal, $H_{8}$ has the maximal Hosoya index.

Let $\mathcal{V}_{9}$ be the set of unicyclic graphs of the form $H_{9}$ (as depicted in Figure 2), where $v_{1}, v_{3}$, and $v_{5}$ are three vertices with $a-1, b-1$, and $c-1$ pendent vertices, $a+b+c=n-3, a, b, c \geq 1$, and one of $a, b, c$ is at least 2. Let $H_{9}^{*}$ be the graph of the form $H_{9}$ satisfying $a, b, c$ almost equal.

Theorem 14. The graph $H_{9}^{*}$ has the maximum Hosoya index among all graphs in $\mathcal{V}_{9}$.

Proof. For $H_{9} \in \mathcal{V}_{9}$, from Lemmas 1 and 2, we have

$$
\begin{aligned}
z\left(H_{9}\right) & =z\left(H_{9}-v_{1}\right)+\sum_{v^{\prime} \in N_{H_{9}}\left(v_{1}\right)} z\left(H_{9}-\left\{v_{1}, v^{\prime}\right\}\right) \\
& =z\left(H_{9}-v_{1}\right)+z\left(H_{9}-\left\{v_{1}, v_{2}\right\}\right) \\
& +z\left(H_{9}-\left\{v_{1}, v_{6}\right\}\right)+(a-1) z\left(H_{9}-\left\{v_{1}, v_{1, a-1}\right\}\right) .
\end{aligned}
$$

For $z\left(H_{9}-v_{1}\right)$, by Lemmas 1 and 2, we have

$$
\begin{aligned}
z\left(H_{9}-v_{1}\right) & =z\left(H_{9}-v_{1}-v_{2}\right) \\
& +\sum_{v^{\prime} \in N_{H_{9}-v_{1}}\left(v_{2}\right)} z\left(H_{9}-v_{1}-\left\{v_{2}, v^{\prime}\right\}\right) \\
& =z\left(H_{9}-v_{1}-v_{2}\right)+z\left(H_{9}-v_{1}-\left\{v_{2}, v_{3}\right\}\right) \\
& =\left[z\left(H_{9}-v_{1}-v_{2}-v_{3}\right)+z\left(H_{9}-v_{1}-v_{2}-\left\{v_{3}, v_{4}\right\}\right)\right. \\
& \left.+(b-1) z\left(H_{9}-v_{1}-v_{2}-\left\{v_{3}, x\right\}\right)\right] \\
& +z\left(H_{9}-v_{1}-\left\{v_{2}, v_{3}\right\}\right) \\
& =[(c+2)+(c+1)+(b-1)(c+2)]+(c+2) \\
& =b c+2 b+2 c+3
\end{aligned}
$$

Here, $x$ is a pendent vertex attached at $v_{3}$.

Observe that $z\left(H_{9}-\left\{v_{1}, v_{2}\right\}\right)=b c+2 b+c+1$,

$$
\begin{aligned}
z\left(H_{9}-\left\{v_{1}, v_{6}\right\}\right) & =z\left(H_{9}-\left\{v_{1}, v_{6}\right\}-v_{5}\right) \\
& +z\left(H_{9}-\left\{v_{1}, v_{6}\right\}-\left\{v_{5}, v_{4}\right\}\right) \\
& +(c-1) z\left(H_{9}-\left\{v_{1}, v_{6}\right\}-\left\{v_{5}, y\right\}\right) \\
& =(b+2)+(b+1)+(c-1)(b+2) \\
& =b c+b+2 c+1
\end{aligned}
$$

where $y$ is one pendent vertex attached at $v_{5}$.

Thus, we obtain

$$
\begin{aligned}
z\left(H_{9}\right) & =z\left(H_{9}-v_{1}\right)+z\left(H_{9}-\left\{v_{1}, v_{2}\right\}\right) \\
& +z\left(H_{9}-\left\{v_{1}, v_{6}\right\}\right)+(a-1) z\left(H_{9}-\left\{v_{1}, v_{1, a-1}\right\}\right) \\
& =(b c+2 b+2 c+3)+(b c+2 b+c+1) \\
& +(b c+b+2 c+1)+(a-1)(b c+2 b+2 c+3) \\
& =a b c+2 a b+2 a c+2 b c+3 a+3 b+3 c+2:=g_{9}(a, b, c) .
\end{aligned}
$$


If $a-b \geq 2$, then we have

$$
\begin{aligned}
& g_{9}(a-1, b+1, c)-g_{9}(a, b, c) \\
& =[(a-1)(b+1) c+2(a-1)(b+1) \\
& +2(a-1) c+2(b+1) c+3(a-1) \\
& +3(b+1)+3 c+2] \\
& -(a b c+2 a b+2 a c+2 b c+3 a+3 b+3 c+2) \\
& =(c+2)(a-b-1) \\
& \geq 0 .
\end{aligned}
$$

Thus, we have $z\left(H_{91}\right)>z\left(H_{9}\right)$.

This implies that, if $H_{9}$ has the maximum Hosoya index, we conclude $|a-c| \leq 1$.

As $b, c$ (resp. $a, c)$ have the same status as shown in Figure 2, we also have $|b-c| \leq 1 .|a-c| \leq 1$. This is the desired result.

Theorem 15. The graph $H_{6}^{*}$ has the maximum Hosoya index among all graphs in $\mathcal{V}_{n}^{4}$ for $n \geq 50$.

Proof. We need only compare $z\left(H_{i}^{*}\right)$ for $1 \leq i \leq 8$.

For $H_{1}^{*}$, as $0 \leq a-b \leq 1,0 \leq a-c \leq 2,0 \leq b-c \leq 1$, we have $b \leq a, c \leq a, c \leq b \leq a$, and $b \geq a-1, c \geq a-2$. Since $a+b+c=n-2$, we get $n-2=a+b+c \geq a+a-1+a-2=3 a-3$, this leads to $a \leq \frac{n+1}{3}$. Therefore,

$$
\begin{aligned}
z\left(H_{1}^{*}\right) & =2 a b c+2 a b+2 a+2 c+2 \\
& \leq 2 a^{3}+2 a^{2}+4 a+2 \\
& \leq \frac{2(n+1)^{3}}{27}+\frac{2(n+1)^{2}}{9}+\frac{4(n+1)}{3}+2:=h_{1}(n)
\end{aligned}
$$

The last inequality holds for a function $f_{1}(a)=2 a^{3}+2 a^{2}+4 a+2$ that is strictly increasing for $2 \leq a \leq \frac{n+1}{3}$.

For $H_{2}^{*}$, we have $0 \leq a-b \leq 2,0 \leq a-c \leq 1,|c-d| \leq 1$. We may assume $0 \leq c-d \leq 1$ without loss of generality. Then, $b \leq a, c \leq a, d \leq c \leq a$, and $b \geq a-2, c \geq a-1, d \geq c-1 \geq a-2$. Thus, $n=a+b+c+d \geq a+a-2+a-1+a-2=4 a-5$, and hence $a \leq \frac{n+5}{4}$. Therefore,

$$
\begin{aligned}
z\left(H_{2}^{*}\right) & =a b c d+a b+a c+a d+c d+1 \\
& \leq a^{4}+4 a^{2}+1 \\
& \leq \frac{(n+5)^{4}}{256}+\frac{(n+5)^{2}}{4}+1:=h_{2}(n) .
\end{aligned}
$$

The last inequality holds for a function $f_{2}(a)=a^{4}+4 a^{2}+1$ that is strictly increasing for $2 \leq a \leq \frac{n+5}{4}$.

For $H_{3}^{*}$, as $a, b, c, d$ are almost equal and $a+b+c+d=n$, we have $a, b, c, d \leq \frac{n+3}{4}$

$$
\begin{aligned}
z\left(H_{3}^{*}\right) & =a b c d+a b+a d+b c+c d+2 \\
& \leq \frac{(n+3)^{4}}{256}+\frac{(n+3)^{2}}{4}+2:=h_{3}(n)
\end{aligned}
$$


For $H_{4}^{*}, 0 \leq a-b \leq 2,|c-d| \leq 1,0 \leq a-c \leq 1$. We may assume $0 \leq c-d \leq 1$ without loss of generality. Then, $b \leq a, c \leq a, d \leq c \leq a$, and $b \geq a-2, c \geq a-1, d \geq c-1 \geq a-2$. Thus, $n-1=a+b+c+d \geq a+a-2+a-1+a-2=4 a-5$, and hence $a \leq \frac{n+4}{4}$. Therefore,

$$
\begin{aligned}
z\left(H_{4}^{*}\right) & =a b c d+a b c+a b d+a c+a d+c d+2 a+c+d \\
& \leq a^{4}+2 a^{3}+3 a^{2}+4 a \\
& \leq\left(\frac{n+4}{4}\right)^{4}+2\left(\frac{n+4}{4}\right)^{3}+3\left(\frac{n+4}{4}\right)^{2}+4 \frac{n+4}{4} \\
& =\frac{(n+4)^{4}}{256}+\frac{(n+4)^{3}}{32}+\frac{3(n+4)^{2}}{16}+n+4:=h_{4}(n) .
\end{aligned}
$$

The last inequality holds for a function $f_{3}(a)=a^{4}+2 a^{3}+3 a^{2}+4 a$ that is strictly increasing for $2 \leq a \leq \frac{n+4}{4}$.

For $H_{5}^{*}, 0 \leq a-b \leq 2,|c-d| \leq 1,0 \leq a-c \leq 1$. We may assume $0 \leq c-d \leq 1$ without loss of generality. Then, $b \leq a, c \leq a, d \leq c \leq a$, and $b \geq a-2, c \geq a-1, d \geq c-1 \geq a-2$. Thus, $n-2=a+b+c+d \geq a+a-2+a-1+a-2=4 a-5$, and hence $a \leq \frac{n+3}{4}$. Therefore,

$$
\begin{aligned}
z\left(H_{5}^{*}\right) & =2 a b c d+a b c+a b d+a b \\
& +2 a c+2 a d+2 c d+2 a+c+d+1 \\
& \leq 2 a^{4}+2 a^{3}+7 a^{2}+4 a+1 \\
& \leq 2\left(\frac{n+3}{4}\right)^{4}+2\left(\frac{n+3}{4}\right)^{3} \\
& +7\left(\frac{n+3}{4}\right)^{2}+4\left(\frac{n+3}{4}\right)+1:=h_{5}(n) .
\end{aligned}
$$

The last inequality holds for a function $f_{4}(a)=2 a^{4}+2 a^{3}+7 a^{2}+4 a+1$ that is strictly increasing for $2 \leq a \leq \frac{n+3}{4}$.

For $H_{6}^{*}, a, b, c, d, e$ are almost equal. Then, $a, b, c, d, e \geq \frac{n-4}{5}$ as $a+b+c+d+e=n$. Therefore,

$$
\begin{aligned}
z\left(H_{6}^{*}\right) & =a b c d e+a b c+a b e+a d e+b c d+c d e \\
& +a+b+c+d+e \\
& \geq\left(\frac{n-4}{5}\right)^{5}+5\left(\frac{n-4}{5}\right)^{3}+5 \frac{n-4}{5}:=h_{6}(n) .
\end{aligned}
$$

For $H_{7}^{*}, a, b, c$ are almost equal. We may assume $a-1 \leq b \leq a+1,0 \leq a-c \leq 1$. Then, we have $b \leq a+1, c \leq a$, and $b \geq a-1, c \geq a-1$. Thus, $n-3=a+b+c \geq a+a-1+a-1=3 a-2$, and hence $a \leq \frac{n-1}{3}$. Therefore,

$$
\begin{aligned}
z\left(H_{7}^{*}\right) & =3 a b c+2 a b+2 b c+3 a+b+3 c+4 \\
& \leq 3 a^{2}(a+1)+2 a(a+1)+2 a(a+1) \\
& +3 a+a+1+3 a+4 \\
& =3 a^{3}+7 a^{2}+11 a+5 \\
& \leq 3\left(\frac{n-1}{3}\right)^{3}+7\left(\frac{n-1}{3}\right)^{2}+11 \frac{n-1}{3}+5:=h_{7}(n) .
\end{aligned}
$$

The last inequality holds for a function $f_{5}(a)=3 a^{3}+7 a^{2}+11 a+5$ which is strictly increasing for $1 \leq a \leq \frac{n-1}{3}$. 
Let $H_{8}^{*}, a, b, c$ are almost equal. We may assume $a-1 \leq b \leq a+1,0 \leq a-c \leq 1$. Then, we have $b \leq a+1, c \leq a$, and $b \geq a-1, c \geq a-1$. Thus, $n-4=a+b+c \geq a+a-1+a-1=3 a-2$, and hence $a \leq \frac{n-2}{3}$. Therefore,

$$
\begin{aligned}
z\left(H_{8}^{*}\right) & =5 a b c+3 a b+3 b c+5 a+2 b+5 c+6 \\
& \leq 5 a^{2}(a+1)+3 a(a+1)+3 a(a+1) \\
& +5 a+2(a+1)+5 a+6 \\
& =5 a^{3}+11 a^{2}+18 a+8 \\
& \leq 5\left(\frac{n-2}{3}\right)^{3}+11\left(\frac{n-2}{3}\right)^{2}+18 \frac{n-2}{3}+8:=h_{8}(n) .
\end{aligned}
$$

The last inequality holds for a function $f_{6}(a)=5 a^{3}+11 a^{2}+18 a+8$ is strictly increasing for $1 \leq a \leq \frac{n-2}{3}$.

For $H_{9}^{*}$, since $a, b, c$ are almost equal, We may assume $a-1 \leq b \leq a+1,0 \leq a-c \leq 1$. Then $b \leq a+1, c \leq a, b \geq a-1, c \geq a-1$. As $n-3=a+b+c \geq a+a-1+a-1=3 a-2$, therefore $a \leq \frac{n-1}{3}$. Thus, we have

$$
\begin{aligned}
z\left(H_{9}^{*}\right)= & a b c+2 a b+2 a c+2 b c+3 a+3 b+3 c+2 \\
& \leq a^{2}(a+1)+2 a(a+1)+2 a^{2}+2 a(a+1) \\
& +3 a+3(a+1)+3 a+2 \\
& =a^{3}+7 a^{2}+13 a+5 \\
& \leq\left(\frac{n-1}{3}\right)^{3}+7\left(\frac{n-1}{3}\right)^{2}+13 \frac{n-1}{3}+5:=h_{9}(n) .
\end{aligned}
$$

The last inequality holds as $f_{7}(a)=a^{3}+7 a^{2}+13 a+5$ is strictly increasing for $1 \leq a \leq \frac{n-1}{3}$. By using the software "Mathematica", we obtain the following comparison.

$$
\begin{aligned}
& h_{2}(n)-h_{1}(n)=\frac{1}{6912}\left(41,889+16,956 n+2706 n^{2}+28 n^{3}+27 n^{4}\right)>0 . \\
& h_{2}(n)-h_{3}(n)=\frac{1}{32}\left(164+81 n+12 n^{2}+n^{3}\right)>0 . \\
& h_{4}(n)-h_{2}(n)=\frac{1}{256}\left(79+140 n+26 n^{2}+4 n^{3}\right)>0 . \\
& h_{6}(n)-h_{4}(n)=\frac{1}{800,000}\left(-13,510,144-1,336,320 n-1,297,840 n^{2}-2040 n^{3}-8245 n^{4}+256 n^{5}\right)>0
\end{aligned}
$$

for $n \geq 37$.

$$
\begin{aligned}
& h_{8}(n)-h_{7}(n)=\frac{1}{27}\left(-70+24 n-9 n^{2}+2 n^{3}\right)>0 \text { for } n \geq 4 . \\
& h_{7}(n)-h_{9}(n)=\frac{1}{27}\left(16-12 n-6 n^{2}+2 n^{3}\right) \geq 0 \text { for } n \geq 4 . \\
& h_{6}(n)-h_{8}(n)=\frac{1}{84,375}\left(-531,148-315 n-67,155 n^{2}-7390 n^{3}-540 n^{4}+27 n^{5}\right)>0 \text { for } n \geq 32 . \\
& h_{6}(n)-h_{5}(n)=\frac{1}{400,000}\left(-6,520,697-793,160 n-730,170 n^{2}-13,520 n^{3}-5685 n^{4}+128 n^{5}\right)>0 \text { for }
\end{aligned}
$$
$n \geq 50$.

The direct computation yields to $z\left(C_{8}\right)=47, z\left(C_{9}\right)=76$.

From the above discussion, we get the result.

\section{Summary and Conclusions}

In this paper, we investigate extremal properties of the famous Hosoya index for unicyclic graphs with diameter at most four. There is no doubt that topological indices have been proven useful for analyzing molecules by means of their graph structure. Especially the Hosoya index is demanding to calculate for general graphs. Thus, special analytical results for the Hosoya index contribute to a better understanding of molecular topology when using this measure. Because of the problem of calculating the Hosoya index efficiently, we also believe that our results can be used for QSAR/QSPR problems. Moreover, the Hosoya index could be calculated on existing drugbanks to determine the value distributions and using them within QSAR/QSPR. Note that this index has a meaningful structural interpretation. As future work, we would like to continue to prove analytical 
results when establishing interrelations between the Hosoya index and known graph measures which have been proven useful for drug design and QSAR/QSPR.

Author Contributions: Conceptualization, W.L., J.B., L.F., F.E.-S. and M.D.; investigation, W.L. and M.D.; data curation, F.E.-S. and T.C.; writing-original draft preparation, M.D.; L.F. and J.B.; writing-review and editing, J.B., L.F., F.E.-S. and M.D.; supervision, J.B., L.F. and M.D.; project administration, M.D.

Funding: This research was supported by NSFC (Nos. 11671402 and 11871479) (L.F. and W.L.), Hunan Provincial Natural Science Foundation (2016JJ2138 and 2018JJ2479) (L.F. and W.L.) and Mathematics and Interdisciplinary Sciences Project of CSU (L.F. and W.L.). NSFC (No. 11701339) (T.C.), the Austrian Science Fund for financial support (P 33001) (M.D.).

Conflicts of Interest: The authors declare no conflict of interest.

\section{References}

1. Basak, S.C. Information-theoretic indices of neighborhood complexity and their applications. In Topological Indices and Related Descriptors in QSAR and QSPR; Balaban, A.T., Devillers, J., Eds.; Gordon and Breach Science Publishers: Amsterdam, The Netherlands, 1999; pp. 563-595.

2. Basak, S.C.; Gute, B.D.; Balaban, A.T. Interrelationship of major topological indices evidenced by clustering. Croat. Chem. Acta 2014, 77, 331-344.

3. Basak S.; Magnuson, V.R. Molecular topology and narcosis. Arzeim. Forsch. Drug Des. 1983, 33, 501-503.

4. Todeschini, R.; Consonni, V.; Mannhold, R. Handbook of Molecular Descriptors; Wiley-VCH: Weinheim, Germany, 2002.

5. Hosoya, H. Topological index. A newly proposed quantity characterizing the topological nature of structural isomers of saturated hydrocarbons. Bull. Chem. Soc. Jpn. 1971, 44, 2332-2339. [CrossRef]

6. Gutman, I.; Hosoya, H.; Uraković, G.; Ristić, L. Two variants of the topological index and the relations between them. Bull. Chem. Soc. Jpn. 1992, 65, 14-18. [CrossRef]

7. Hou, Y. On acyclic systems with minimal Hosoya index. Discret. Appl. Math. 2002, 119, 251-257. [CrossRef]

8. Liu, H. The proof of a conjecture concerning acyclic molecular graphs with maximal Hosoya index and diameter 4. J. Math. Chem. 2008, 43, 1199-1206. [CrossRef]

9. Wagner, S. Extremal trees with respect to Hosoya index and Merrifield-Simmons index. MATCH Commun. Math. Comput. Chem. 2007, 57, 221-233.

10. Deng, H.; Chen, S. The extremal unicyclic graphs with respect to Hosoya index and Merrifield-Simmons index. MATCH Commun. Math. Comput. Chem. 2008, 59, 171-190.

11. Hua, H. Hosoya index of unicyclic graphs with prescribed pendent vertices. J. Math. Chem. 2008, 43, 831-844. [CrossRef]

12. Ou, J. On extremal unicyclic molecular graphs with prescribed girth and minimal Hosoya index. J. Math. Chem. 2007, 42, 423-432. [CrossRef]

13. $\mathrm{Xu}, \mathrm{K} . ; \mathrm{Xu}, \mathrm{B}$. Some extremal unicyclic graphs with respect to Hosoya index and Merrifield-Simmons index. MATCH Commun. Math. Comput. Chem. 2009, 62, 629-648.

14. Yu, G.; Feng, L.; Ilić, A. The largest $n-1$ Hosoya indices of unicyclic graphs. Filomat 2016, 30, $2573-2581$. [CrossRef]

15. Deng, H. The largest Hosoya index of $(n, n+1)$-graphs. Comput. Math. Appl. 2006, 56, 2499-2506. [CrossRef]

16. Wagner, S.; Gutman I. Maxima and minima of the Hosoya index and the Merrifield-Simmons index: A survey of results and techniques. Acta Appl. Math. 2010, 112, 323-346. [CrossRef]

17. Bollobás, B. Modern Graph Theory; Springer: New York, NY, USA, 1998. 